\title{
DePRESSÃO NO HOSPITAL GERAL: ESTUdO DE 136 CASOS
}

Renério fráguas júnior*, tânia Correa de Toledo Ferraz Alves

Trabalho desenvolvido no grupo de interconsultas do Instituto de Psiquiatria do Hospital da Clínicas da Faculdade de Medicina da Universidade de São Paulo, SP.

RESUMO - Objetivo. 0 objetivo deste estudo é investigar os sintomas depressivos que diferenciam pacientes com e sem depressão associada a condiçöes médicas.

Métodos. Foram estudados 293 pedidos de interconsulta à psiquiatria, consecutivos, no ano 1998, sendo $168(57,5 \%)$ mulheres e 124 (42,5\%) homens, com idades variando de 18 a 93 anos $(47,2+/-18,0$ anos). 0 diagnóstico psiquiátrico foi realizado através de uma entrevista clínica aberta, utilizando-se os critérios do DSMIV. A análise estatística foi realizada através dos testes de Quiquadrado e regressão logística.

Resultados. Dos 293 pacientes avaliados, 230 (78,50\%) preenchiam critérios para diagnóstico psiquiátrico; sendo que $136(59,1 \%)$ apresentaram transtornos dentro do espectro depressivo: Depressão Maior $(n=60-26,1 \%)$, Depressão Menor $(n=31-13,5 \%)$, Depressão Secundária $(n=19-8,3 \%)$ e Reação de Ajustamento com Humor Depressivo ( $n=26-11,3 \%$ ). À exceção do aumento de apetite, aumento de peso, agitação e ilusōes, a ocorrência ou não de depressão foi significativamente diferente $(p<0,05)$ quando se comparou a presença com a ausência dos demais sintomas potencialmente depressivos. A anedonia e a piora matinal só foram detectadas em, respectivamente, 4 e 3 pacientes sem depressão, 0 que não permitiu sua inclusão na análise de regressão logística. De acordo com a análise de regressão logística (IC=95\%), as variáveis selecionadas para explicar o diagnóstico de depressäo foram: pensamento de morte $(O R=20,6 ; 2,5-170,5)$, irritabilidade $(O R=4,5 ; \quad I, 7-11,9)$, despertar precoce $(O R=15,0 ; \quad I, 7-129,3)$ e perda de peso (OR=8,I; 2,6-24,4).

Conclusão. Pensamentos de morte, irritabilidade, despertar precoce, perda de peso anedonia e piora matinal foram os sintomas que mais fortaleceram 0 diagnóstico de depressão. Mesmo sintomas que poderiam ser manifestação da condiçāo clínica e/ou da depressão (tais como insônia, diminuição da concentração, fadiga e lentificaçäo) foram significativamente mais associados ao diagnóstico da depressão. Estes dados reforçam a necessidade do clínico investigar ativamente 0 diagnóstico de depressão na presença de sintomas físicos que podem ser decorrentes de uma condição médica geral ou de uma depressão. Devido a sua elevada prevalência, a depressão não pode ser um diagnóstico de exclusão.

UNITERMOs: Depressão. Hospital Geral. Interconsulta psiquiátrica.

\section{INTRODUÇÃO}

Transtornos depressivos determinam um grande prejuízo na esfera pessoal e familiar, acometem principalmente adultos jovens entre 20 e 30 anos de idade, atingindo duas vezes mais mulheres que homens. O National ComorbiditySurveyrelatou uma prevalência de depressão maior ao longo da vida de $17,1 \%$ '. Esta prevalência de transtornos depressivos é ainda mais elevada na presença de condições médicas, tanto em estudos nacionais como internacionais, variando de $12 \%$ a $83 \%^{2-6}$.

A depressão associada a outras doenças não psiquiátricas é, em geral, subdiagnosticada ${ }^{7,8}$ por diversos fatores ${ }^{9}$, dentre os quais, ressaltamos a dificuldade em se identificar os sintomas depressivos.

\footnotetext{
*Correspondência: Instituto Psquiatria do $\mathrm{HC}$

R. Ovidio Pires de Campos, s/n-05403-010

S.Paulo-SP-E-mail: rfraguas@henet.usp.br
}

Diversos autores ${ }^{5,10-13}$ demonstraram que as características psicopatológicas da depressão nos pacientes com doença clínica são diferentes daquelas encontradas em pacientes com depressão primariamente psiquiátrica. Isto decorre, provavelmente, devido a fatores emocionais, físicos e psicológicos diretamente ligados à doença clínica à qual a depressão está associada. Além disso, alguns sintomas depressivos como insônia, diminuição da concentração, inapetência, emagrecimento e fraqueza são encontrados em outras doenças não psiquiátricas e podem ser atribuídos exclusivamente a estas, e o diagnóstico de depressão não é realizado.

O diagnóstico precoce e tratamento é essencial, uma vez que além do comprometimento da qualidade de vida, a depressão acarreta significativo aumento da morbidade e mortalidade decorrentes da condição médical ${ }^{14}$. Dentre os vários comprometimentos já descritos, a depressão pode aumentar o tem- po de internação ${ }^{15}$, aumentar o risco de taquicardia ventricular ${ }^{10}$, aumentar a mortalidade no pós-operatório ${ }^{16}$ ou após o infarto agudo do miocárdio ${ }^{17}$, diminuir a adesão ao tratamento e à reabilitação ${ }^{2}$, e, em cardiopatas, constituir um dos principais fatores de risco para complicações cardíacas ${ }^{18-27}$.

\section{Métodos}

O objetivo do nosso estudo é investigar os sintomas da depressão associada a condições médicas no Hospital das Clínicas da Faculdadede Medicina da Universidade de São Paulo (HCFMUSP) e identificar aqueles que diferenciam pacientes comesem depressão. Foram estudados, de modo consecutivo, 293 casos atendidos, no ano de 1998, pelo Grupo de Interconsultas do Instituto de Psiquiátrica do HCFMUSP. OGrupo prestaassistência a todas as clínicas (ex. neurologia, ortopedia, reumatologia cardiologia, cirurgia) do HCFMUSP. Os pacientes são avaliadosmediantesolicitaçãodeinterconsulta psiquiá- 
FRÁGUAS JÚNIOR R ET AL.

trica por parte dos médicos das diversas clínicas. Oatendimento de cada caso foirealizado por um residente do segundo ano, supervisionado por ummédico assistente.

Dos pacientes avaliados, $168(57,5 \%)$ eram mulheres e 124 (42,5\%) homens. As idades variaram de 18 a 93 anos $(47,21+/-18,05$ anos). Noventaedois (33,6\%) pacienteseram casados, $33(12 \%)$ separados, I08 (39,4\%) solteiros e 4 I (I5\%) viúvos.

Para cada caso foi preenchido um protocolo de registro de dados que é parte da rotina assistencial do Grupo de Interconsultas. Este protocolo possui itens relativos a características psicopatológicas da depressão maior, melancólica ou não. Cada sintoma potencialmente depressivo ou depressivo-melancólico foi especificado de acordo com o julgamento do psiquiatra interconsultor, se decorrente da depressão, de outra condição psiquiátrica ou de outra condição médica não psiquiátrica. A classificação do sintoma se melancólico ou não, foi definida "a priori", de acordo com os critérios do DSM-IV. O protocolo também possui o registro do motivo do pedido de interconsulta, e a concordância do clínico com a orientação psiquiátrica.

O diagnóstico psiquiátrico foi realizado através de uma entrevista clínica aberta, utilizando-se todos os dados disponíveis (i.e. exame físico, exames de laboratório, discussão com o médico do hospital geral com a psicóloga, familiares e enfermagem) e de acordo com os critérios do DSM-IV.

\section{ANálSE ESTATISTICA}

Os sintomas potencialmente depressivos foram analisados de modo descritivo de acordo com o julgamento do psiquiatra em relação à sua origem, se decorrentes de depressão, de outros transtornos psiquiátricos ou de outros transtornos médicos. Os sintomas foram considerados no seu aspecto qualitativo, ou seja, se presentes ou ausentes.

A distribuição da presença ou não de depressão em relação a cada sintoma potencialmente depressivo, independente da avaliação prévia do residente se aquele sintoma era secundário à depressão ou a outras condições, foi analisada através do teste do qui-quadrado. A significância esta-

Gráfico I - Motivo do encaminhamento referido no pedido de consulta das diversas clínicas do HCFMUSP para a psiquiatria no ano 1998

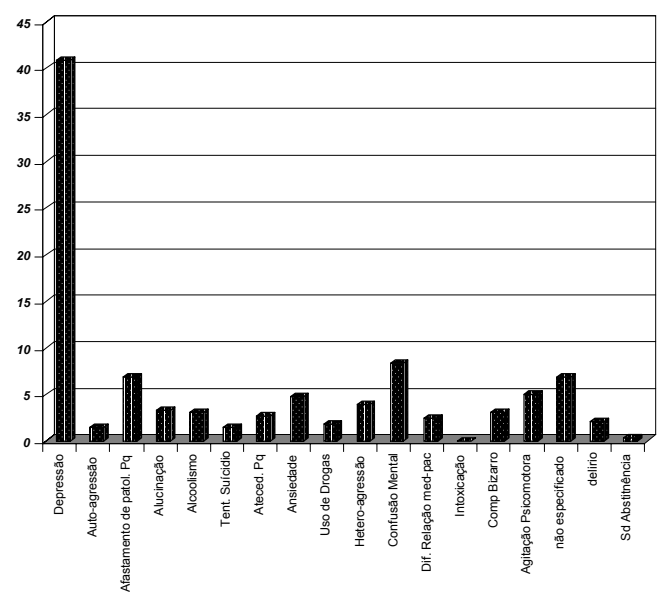

Afastamento de pato I.Pq:afastamento de patologia psiquiátrica

Tent. Suicídio: tentativa desuicídio

Anteced.Pq: antecedentede distúrbio psiquiátrico

Dif. Relação med-pac: dificuldade da relação entre médico e paciente Comp. Bizarro: comportamento bizarro

Sd.Abstinência: síndromedeabstinência

Gráfico 2 - Distribuição dos diagnósticos psiquiátricos realizados a partir dos pedidos de consulta do Hospital Geral do HCFMUSP no ano 1998

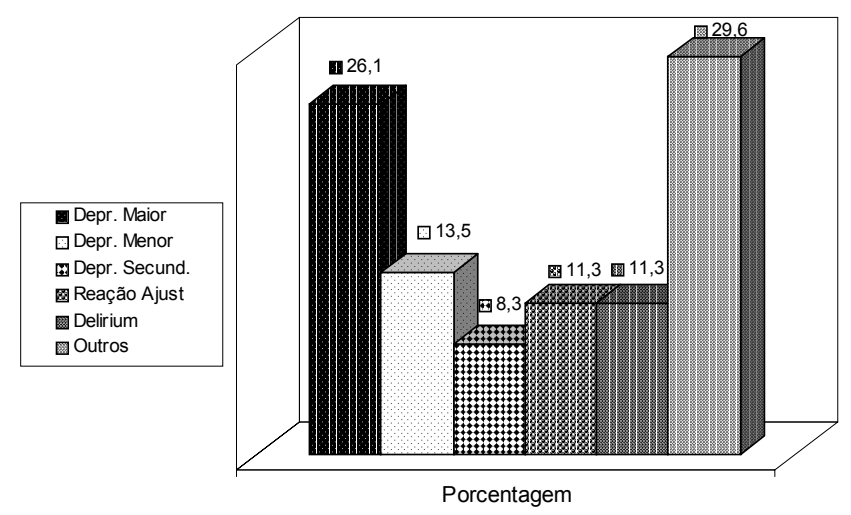

Depr.Maior: depressãomaior

Depr.Menor: depressãomenor

Depr.Secund.: depressãoseciundária

ReaçãoAjust. Reaçãoajustamento

tística foi de 0,05 . Para a avaliação dos sintomas que mais contribuíram para 0 diagnóstico de depressão foi realizada a análise de regressão logística, utilizando-se os fatores que apresentaram nível de significância menor que 0,25 na análise univariada e aplicando-se o processo de seleção de variáveis "stepwise". 
Tabela I - Distribuição dos sintomas potencialmente melancólicos de acordo com sua ausência, presença devido à depressão ou presença devido a outras condiçōes

\begin{tabular}{lccc}
\hline & AUSENTE & PRESENTE POR & PRESENTE PELA \\
OUTRASCAUSAS & DEPRESSÄO \\
Culpa & & $10(3,9 \%)$ & $35(13,5 \%)$ \\
Anedonia & $214(82,6 \%)$ & $12(4,7 \%)$ & $83(32,3 \%)$ \\
Despertarprecoce & $162(63,0 \%)$ & $9(3,6 \%)$ & $50(19,8 \%)$ \\
Pioramatinal & $194(76,7 \%)$ & $8(3,2 \%)$ & $38(15,1 \%)$ \\
Humornãoreativo & $205(81,7 \%)$ & $16(6,4 \%)$ & $24(9,6 \%)$ \\
Perdade peso & $211(84,1 \%)$ & $16(6,5 \%)$ & $31(12,5 \%)$ \\
Humordiferente & $174(81,0 \%)$ & $16(6,5 \%)$ & $31(12,5 \%)$ \\
Perdadeapetite & $201(81,0 \%)$ & $27(10,5 \%)$ & $42(16,4 \%)$ \\
Agitação & $187(73,0 \%)$ & $19(7,4 \%)$ & $15(5,8 \%)$ \\
Lentidão & $223(86,6 \%)$ & $21(8,0 \%)$ & $34(14,6 \%)$ \\
\hline
\end{tabular}

Tabela 2 - Distribuiçáo dos sintomas potencialmente depressivos de acordo com sua ausência, presença devido à depressão ou presença devido a outras condiçōes

\begin{tabular}{lccc}
\hline & AUSENTE & PRESENTE POR & PRESENTE PELA \\
OUTRAS CAUSAS & DEPRESSÄO \\
Insôniainicial & $152(59,6 \%)$ & $10(3,9 \%)$ & $35(13,5 \%)$ \\
Insôniaintermedíaria & $199(80,9 \%)$ & $18(7,3 \%)$ & $29(11,8 \%)$ \\
Insôniaterminal & $192(75,9 \%)$ & $23(9,1 \%)$ & $38(15,0 \%)$ \\
Diminuiçãaconcentração & $144(55,4 \%)$ & $47(18,1 \%)$ & $69(26,5 \%)$ \\
Aumentoapetite & $244(94,9 \%)$ & $7(2,7 \%)$ & $6(2,3 \%)$ \\
Aumentopeso & $238(91,5 \%)$ & $11(4,2 \%)$ & $11(4,2 \%)$ \\
Pensamentosuicida & $205(79,5 \%)$ & $18(7,0 \%)$ & $35(13,6 \%)$ \\
Fadiga & $114(42,9 \%)$ & $60(22,6 \%)$ & $92(34,6 \%)$ \\
PensamentoMorte & $178(69,0 \%)$ & $22(8,5 \%)$ & $58(22,5 \%)$ \\
Irritabilidade & $175(67,8 \%)$ & $34(13,2 \%)$ & $49(19,0 \%)$ \\
llusóes & $226(89,3 \%)$ & $18(7,1 \%)$ & $9(3,6 \%)$ \\
\hline
\end{tabular}

\section{REsUltAdos}

Os motivos mais freqüentes dos pedidos de interconsulta foram: depressão, afastar patologia psiquiátrica e presença de antecedente psiquiátrico (Gráfico I).

Dos 293 pacientes, 230 (78,50\%) preenchiam critérios para diagnóstico psiquiátrico segundo o DSM-IV. Dos 230 casos com diagnóstico psiquiátrico, I36 (59, I\%) apresentaram transtornos dentro do espectro depressivo, assim distribuídos: Depressão Maior: 60 pacientes (26,1\%), Depressão Menor: 31 pacientes (13,5\%), DepressãoSecundária: 19 pacientes $(8,3 \%)$, Reação de Ajustamento com Humor Depressivo: 26 pacientes (II,3\%). Delirium foi diagnosticado em 26 pacientes $(\mathrm{II}, 3 \%)$ e 68 pacientes $(29,6 \%)$ apresentaram outros diagnósticos (Gráfico 2).

Em relação à sintomatologia no momento da avaliação, os sintomas potencialmente depressivos-melancólicos mais freqüentemente atribuídos à depressão pelo psiquiatra interconsultor foram: anedonia $(n=83-32,3 \%)$ e despertar precoce $(n=50-19,8 \%)$ (Tabela I). Os sintomas potencialmente depressivos não-melancólicos mais freqüentemente atribuídos à depressão foram: fadiga ( $n=92-34,6 \%$ ) e diminuição da concentração ( $n=69-26,5 \%$ ) (Tabela 2).

Dentre os sintomas potencialmente depressivos-melancólicos, perda de peso $(n=39-15,2 \%)$ e perda de apetite $(N=27-$ $10,5 \%)$ foram os mais freqüentemente atribuídos a outras condições médicas pelo psiquiatra interconsultor (Tabela I). Os sintomas potencialmente depressivos que mais freqüentemente foram identificados como secundáriosa outras condições médicas foram a fadiga ( $n=60-22,6 \%)$ e a diminuição da concentração $(n=47-18,1 \%)$ (Tabela 2).
Àexceção do aumento de apetite, aumento de peso, agitação eilusões, o diagnóstico de depressão apresentou distribuição significativamente diferente em relação à presença ou não dos sintomas depressivos (Tabelas 3 e 4 ).

De acordo com a análise de regressão logística, as variáveis selecionadas para explicar o diagnóstico de depressão foram: pensamento de morte $(O R=20,6, I C=95 \%, 2,5$ $170,5)$, irritabilidade $(O R=4,5, I C=95 \%$, $I, 7-I I, 9)$, despertar precoce $(O R=15,0$, $\mathrm{IC}=95 \%, \quad I, 7-129,3)$ e perda de peso $(\mathrm{OR}=8, \mathrm{I}, \mathrm{IC}=95 \%, 2,6-24,4)$. As variáveis anedonia e piora matinal não entraram no modelo pelo reduzido número de pacientes sem depressão que apresentavam esses sintomas, respectivamente $4 \mathrm{e} 3$ pacientes. Aumento do apetite também foi excluído do modelo, pois estava presente em apenas seis pacientes com depressão e em quatro pacientes com outros diagnósticos.

\section{Discussão}

Nossos achados revelaram uma grande prevalência de transtornos dentro do espectro depressivo. Transtornos depressivos foram diagnosticados em I 36 pacientes (46,4 I\% dos pedidos). Depressão maior foi o diagnóstico mais freqüente, realizado em 60 (44, I2\%) pacientes, depressão menor foi diagnosticada em 3 I pacientes (22,79\%), depressão secundária a doenças e medicamentos em I9 (8,3\%) e a reação de ajustamento com humor depressivo em 26 pacientes (II,3\%) (Gráfico 2). A semelhança entre sintomas da depressão e sintomas de outras condições médicas dificulta o diagnóstico de depressão. Vários autores vêm enfocando o assunto visando facilitar e dar confiabilidade ao diagnóstico da depressão no contexto médico.

Uma linha de pesquisa investigou sintomas que caracterizassem pacientes com depressão mais grave, assim assegurando que aquela sintomatologia era depressiva e não decorrente da condição médica. Investigando os sintomas mais freqüentes em pacientes com pontuação acima de 13 no inventário de Beck para depressão $0^{28}$,Scwab et al. (1967) encontraram culpa, pessimismo, sentimento de fracasso e insatisfação com a imagem corporal. Moffic e Payke ${ }^{29}$ encontraram culpa, pessimismo, tristeza, preocupação somática, irritabilidade, ideação suicida, anorexia e insônia; Plumb e 
Fráguas JÚNIOR R ET AL.

Tabela 3 - Distribuição do diagnóstico de depressão ou não-depressão de acordo com os sintomas potencialmente depressivos

\begin{tabular}{llccc}
\hline Sintomas & & Depressäo presente & Depresente ausente & p \\
Insôniainicial & Ausente & $50(45 \%)$ & $61(55 \%)$ & 0,001 \\
& Presente & $75(79 \%)$ & $20(21 \%)$ & \\
Insôniaintermediária & Ausente & $84(54 \%)$ & $72(46 \%)$ & 0,003 \\
& Presente & $31(79 \%)$ & $9(21 \%)$ & \\
Insôniaterminal & Ausente & $78(52 \%)$ & $72(48 \%)$ & 0,001 \\
& Presente & $44(81 \%)$ & $10(19 \%)$ & \\
Diminuiçäoconcentração & Ausente & $55(51 \%)$ & $53(49 \%)$ & 0,005 \\
& Presente & $72(70 \%)$ & $31(30 \%)$ & \\
Aumentoapetite & Ausente & $117(59 \%)$ & $80(41 \%)$ & 1 \\
& Presente & $6(60 \%)$ & $4(40 \%)$ & \\
Aumentopeso & Ausente & $114(60 \%)$ & $76(40 \%)$ & 0,789 \\
& Presente & $12(63 \%)$ & $7(37 \%)$ & \\
Ideaçãosuicida & Ausente & $88(53 \%)$ & $77(47 \%)$ & 0,001 \\
& Presente & $37(88 \%)$ & $5(12 \%)$ & \\
Fadiga & Ausente & $30(35 \%)$ & $55(65 \%)$ & 0,001 \\
\multirow{3}{*}{ Pensamentodemorte } & Presente & $103(78 \%)$ & $29(22 \%)$ & \\
& Ausente & $65(46 \%)$ & $76(54 \%)$ & 0,001 \\
Irritabilidade & Presente & $60(90 \%)$ & $7(10 \%)$ & \\
& Ausente & $70(51 \%)$ & $67(49 \%)$ & 0,001 \\
Ilusõos & Presente & $54(76 \%)$ & $17(24 \%)$ & \\
& Ausente & $108(60 \%)$ & $72(40 \%)$ & 0,35 \\
& Presente & $12(50 \%)$ & $12(50 \%)$ & \\
\hline
\end{tabular}

Tabela 4 - Distribuição do diagnóstico de depressão ou näo-depressão de acordo com os sintomas potencialmente melancólicos

\begin{tabular}{llccc}
\hline DEPRESSÃO & & AUSENTE & PRESENTE & P \\
Culpa & Ausente & $93(54 \%)$ & $78(46 \%)$ & 0,001 \\
& Presente & $35(92 \%)$ & $3(8 \%)$ & \\
Anedonia & Ausente & $47(39 \%)$ & $75(61 \%)$ & 0,001 \\
& Presente & $82(95 \%)$ & $4(5 \%)$ & \\
Despertar precoce & Ausente & $74(49 \%)$ & $77(51 \%)$ & 0,001 \\
& Presente & $48(91 \%)$ & $5(9 \%)$ & \\
Pioramatinal & Ausente & $84(52 \%)$ & $77(48 \%)$ & 0,001 \\
& Presente & $38(93 \%)$ & $3(7 \%)$ & \\
Humornãoreativo & Ausente & $93(56 \%)$ & $72(44 \%)$ & 0,003 \\
& Presente & $30(83 \%)$ & $6(17 \%)$ & \\
Perdadepeso & Ausente & $66(48 \%)$ & $71(52 \%)$ & 0,001 \\
\multirow{4}{*}{ Humordiferente } & Presente & $59(86 \%)$ & $10(14 \%)$ & \\
& Ausente & $82(53 \%)$ & $73(47 \%)$ & 0,001 \\
Anorexia & Presente & $37(84 \%)$ & $7(16 \%)$ & \\
& Ausente & $77(54 \%)$ & $66(46 \%)$ & 0,002 \\
Agitação & Presente & $48(76 \%)$ & $15(24 \%)$ & \\
& Ausente & $106(60 \%)$ & $70(40 \%)$ & 0,652 \\
Lentificação & Presente & $20(65 \%)$ & $11(35 \%)$ & \\
& Ausente & $92(56 \%)$ & $72(44 \%)$ & 0,001 \\
& Presente & $39(83 \%)$ & $8(17 \%)$ & \\
\hline
\end{tabular}

Holland ${ }^{30}$ encontraram baixa auto-estima como o principal indicador de depressão em pacientes com câncer. Clark et al ${ }^{31}$ relataram que os sintomas que melhor discriminavam a depressão mais grave eram: sentimento de fracasso, de punição, ideação suicida e choro freqüente. Uma limitação deste tipo de estudo é que não considera pacientes com transtorno depressivo leve ou mesmo a depressão subsindrômica que podem apresentar psicopatologia depressiva diferente, e segundo Williams et a $\left.\right|^{32}$, a depressão subsindrômica é mais freqüente do que a depressão maior em clínicas de atenção primária e se associa a significativo comprometimento funcional.

Em nossa casuística, fadiga e diminuição da concentração foram os sintomas depressivos não-melancólicos mais freqüentemente atribuídos à depressão, respectivamente em 92 (34\%) e 69 (26,5\%) pacientes com diagnóstico de depressão (Tabela 2). Entretanto, estes sintomas também foram os mais freqüentemente atribuídos a outras condições médicas, fadiga em 60 pacientes $(22,6 \%)$ e diminuição da concentração em 47 pacientes (I8, I\%) (Tabela 2). Aelevada frequiência de dificuldade para se concentrar e a diminuição da energia em pacientes com depressão associada a outras condições médicas já foi relatada por Koenig ${ }^{5}$. Por outro lado, os trabalhos acima descritos que investigaram a psicopatologia dos quadros mais graves de depressão utilizando a escala de Beck ${ }^{28-31}$ relataram basicamente sintomas psicológicos, sendo que fadiga não foi relatada em nenhum destes estudos, e a escala de Beck não investiga diminuição da concentração. Embora diante de um paciente com fadiga ou com diminuição da concentração, em nossa casuística, a distribuição do diagnóstico de depressão e não-depressão fosse significativamente diferente (fadiga, $p=0,00 \mathrm{I}$; diminuição da concentração, $p=0,005$ )(Tabela 3), estes sintomas não foram selecionados pelo modelo da análise de regressão logística, o que diminui o valor destes como marcadores de depressão. Concordamos com Cavanaugh ${ }^{13}$ quando afirma que a fadiga, além de alterações do sono, peso, apetite e da psicomotricidade, ajudam a corroborar o diagnóstico quando em excesso ou associados à sintomas cognitivose afetivos da depressão. Da mesma maneira, em relação à diminuição de concentração, acreditamos que outros sintomas depressivos devam corroborar o diagnóstico, uma 
vez que o delirium também comum no hospital geral, cursa com diminuição da concentração, com labilidade afetiva e com freqüência é considerado como depressão por médicos não psiquiatras ${ }^{33}$.

Os sintomas melancólicos mais freqüentemente atribuídos à depressão foram anedonia e despertar precoce, presentes respectivamente em 83(32,3\%) e 50 (19,8\%) pacientes. Adistribuição do diagnóstico de depressão ou não-depressão foi significativamente diferente nestes dois sintomas (anedonia, $p=0,00 \mathrm{I}$; despertar precoce, $\mathrm{p}=0,00 \mathrm{I})(\mathrm{Ta}-$ bela 4). A anedonia estava presente em apenas quatro pacientes sem o diagnóstico de depressão, o que não permitiu sua inclusão no modelo de regressão logística. No estudo de Furlaneto ${ }^{34}$, anedonia também foi um dos sintomas que melhor discriminaram depressão de não-depressão em pacientes em enfermaria de hospital geral. Wooley et a $\left.\right|^{35}$ relataram que a investigação da anedonia, além do humor depressivo, foi suficiente para a detecção de casos de depressão em uma Unidade de Urgências. Considerando estes dados, concluímos que a anedonia é um bom marcador de depressão. A investigação de anedonia tornase fundamental, particularmente em idosos, que de acordo com Gallo et a ${ }^{36}$, podem apresentar depressão sem tristeza.

$A$ análise de regressão logística revelou que 0 despertar precoce $(O R=15,0$, $I C=95 \%, I, 7-\mid 29,3)$, pensamento de morte $(\mathrm{OR}=20,6, I C=95 \%, 2,5-170,5)$, irritabilidade $(O R=4,5, I C=95 \%, I, 73-I I, 9)$ e perda de peso $(O R=8, I, I C=95 \%, 2,6-24,4)$ foram os sintomas que mais explicaram o diagnóstico de depressão.

Nossos dados reforçam a importância da investigação de sintomas melancólicos (despertar precoce, perda de peso e anedonia), de modo sistemático em pacientes no hospital geral para se detectar a depressão. Além dos sintomas melancólicos, irritabilidade $\mathrm{e}$ pensamentos de morte também contribuíram para o diagnóstico de depressão de acordo com a análise de regressão logística. Diante de um paciente com irritabilidade, a distribuição do diagnóstico de depressão ou nãodepressão foi significativamente diferente $(p=0,00 \mathrm{I})$ (Tabela 3). Segundo Schwab et al. ${ }^{37}$ e Cavanaugh ${ }^{11,12}$, irritabilidade, além de choro e tristeza, seriam freqüentes em pa- cientes no hospital geral, mesmo sem depressão, e só deveriam ser considerados como sintomas depressivos se intensos ou graves. De acordo com o estudo de Moffice Payke ${ }^{29}$, acima citado, irritabilidade foi um dos sintomas mais freqüentes em pacientes com Beck > 13. Nossos achados reforçam o valor da irritabilidade como um sintoma da depressão no hospital geral.

Cavanaugh ${ }^{13}$ propõe a modificação dos critérios diagnósticos do DSM-IV, adaptandoos para os pacientes internados em unidades clínicas. A autora propõe a inclusão da falta de esperança ou apatia como alternativa ao humor depressivo e anedonia. Além disso, Cavanaugh salienta a falta de interesse por pessoas (familiares, pacientes, equipe) por parte do paciente como indicativo de depressão. Nossos achados evidenciam que a anedonia deve servalorizada na investigação da depressão no contexto médico, ressalte-se que a falta do prazer em estar com pessoas seja por nós considerada anedonia. Embora Cavanaugh também inclua irritabilidade quando intensa como um possível sintoma depressivo, e embora seja um sintoma psicológico, este não foi significativamente valorizado por Cavanaugh no que diz respeito a auxiliar a realização do diagnóstico de depressão no contexto médico.

Nossa casuística ressalta a relevância da depressão em causar o emagrecimento. Mesmo que a perda de peso possa ser decorrente de outras doenças, este foi um sintoma sele cionado pela análise de regressão logística para explicar o diagnóstico de depressão. Defendemos a conduta de que diante de um paciente com emagrecimento, mesmo com diagnóstico de outra doença que possa causar a perda de peso, o diagnóstico de depressão deva ser descartado devido à sua elevada comorbidade. Do mesmo modo, se o despertar precoce for atribuído de modo generalizado ao estresse decorrente da hospitalização e das rotinas hospitalares, o diagnóstico de depressão poderá não ser realizado.

\section{ConClusão}

De acordo com os dados desta pesquisa, pensamentos de morte, irritabilidade, despertar precoce e perda de peso foram os sintomas que melhor explicaram o diagnóstico de depressão. A presença de anedonia e piora matinal fortalecem o diagnóstico da depressão, pois raramente foram encontradas na ausência desta. Mesmo outros sintomas como insônia, diminuição da concentração, fadiga e lentificação, que poderiam ser manifestação da condição clínica e não da depressão, nesta casuística, foram significativamente mais associados ao diagnóstico da depressão do que com a ausência desta.

Estes dados reforçam a necessidade do clínico de investigar ativamente o diagnóstico de depressão na presença de sintomas físicos que podem ser decorrentes de uma condição médica geral ou de uma depressão. Devido a sua elevada prevalência, a depressão não pode ser um diagnóstico de exclusão.

\section{SUMMARY \\ Depression in General Hospital: a STUDY OF 136 CASES}

OBJECTIVE. To investigate the depressive symptoms that differentiate patients with and without depression associated to medical disease.

MethoD. During 1998, 293 consecutive referrals for consultations were studied, 168 (57.5\%) of females and I24 (42.5\%) males, with ages ranging from 18 to 93 years $(47.2 \pm$ 18.0 years). Psychiatric diagnosis was made by clinical interviews, according to DSM-IV criteria. For statisticalanalysis the chi-square and logistic regression testswereadopted.

RESULTS. Of the 293 patients under study, $230(78.5 \%)$ fulfilled the criteria for psychiatric diagnosis, 136 (59.1\%) of them presented disorders within the depressivespectrum:Major Depression ( $n=60-26.1 \%$ ), MinorDepression $(n=31-13.5 \%)$. Secondary Depression $(n=$ 19-8.3\%) and Adjustment Disorder with Depressive Mood ( $n=26-1 / .3 \%)$. Excep for greater appetite, weight gain, agitation and delusions, theoccurrence ornot of the diagnostic of depression was significantly different $(p<0.05)$ when comparingthepresence with the absence of the remaining potential depressive symptoms. Theanedoniaandmorningworsening were detected in 4 and 3 patients without depression respectively, which did notallow the inclusion of these symptoms in the regression analysis model. In accordance with the logistic regression analysis (IC=95\%) the selected variables to explain diagnosis of depression were thoughts of death $(O R=20.6 ; 2.5-170.5)$, irritability $(O R=4.5 ; \quad 1.7-I I .9)$, early 
Fráguas JÚNIOR R et AL.

awakening $(O R=15.0 ; 1.7-129.3)$ and weight loss $(O R=8.1 ; 2.6-24.4)$.

CONCLUSION. Thoughts of death, irritability, earlyawakening, loss of weight, anhedonia (loss of pleasure); and morning worsening were the symptoms that strongly supported depression diagnosis. Even symptoms that could be a manifestation of medical condition and/or of depressions (such as sleeplessness, diminished concentration, fatigue and slowing down) were significantly more associated to the diagnosis of depression. These data outline the need for an active clinical investigation of depression by the physician in the case of physical symptoms that could be related to a medical condition or a depression. Owing to its high prevalence, the diagnosis of depression should not be made on an exclusion basis. [Rev Assoc Med Bras 2002; 48(3): 225-30 ]

Key Words: Depression. General Hospital. Psychiatric consultation.

\section{REFERÊNCIAS}

I. Kessler RC, McGonagle KA, Zhao S, Nelson $C B$, Hughes M, Eshleman S, Wittchen HU, Kendler JS. Lifetime and I 2-month prevalence of DSM-III-R psychiatric disorders in the United States. Arch Gen Psych 1 994;5 I:8- 19.

2. Botega NJ, Bio MC, Zomignani MA, Garcia CJr, Pereira WAB. Transtornos do humor em enfermaria de clínica médica e validação de escala de medida (HAD) de ansiedade e depressão. Rev Saúde Publica 1995; 29(5):355-63.

3. Martucci M, Balestrieri M, Bisoffi G et al . Evaluation psychiatric morbidity in a general hospital: a two-phase epidemiological survey. Psychol Med I 999;29(4):823- 32.

4. Pearson SD, Katzelnick DJ, Simon GE et al. Depression among high utilizers of medical care. J Gen Intern Med 1999; I 4(8):46I-8.

5. Koenig HG, Pappas P, Holsinger T. Assessing diagnostic approaches to depression in medically ill older adults: how reliably can mental health professionals make judgment about the cause of symptoms? J Am Geriatr Soc 1995;43: 472-8.

6. Hasket RF. Diagnostic categorization of psychiatric disturbance in Cushing's syndrome. JPsychiatry 1985; I 42:91।-16.

7. Badger LW, deGruy FV, Hartman J et al. Patient presentation, interview content, and the detection of depression by primary care physicians. Psychosom Med 1994; 56: I 28-35.
8. Fráguas JrR, Ramadan ZBA, Pereira ANE, Wajngarten $M$. Depression with irritability in patients undergoing coronary artery bypass graft surgery: the cardiologist's role. Gen Hosp Psychiatry 2000;22:365-374.

9. Robbins JM, Laurence KJ, Cathébras P, Yaffe MJ, Dworkind M. Physician characteristics and the recognition of depression and anxiety in primary care. Med Care 1994;32:795-8I 2.

10. Carney MC, Freedkabd KE, Rich MW, Smith LJ, Jaffe AS. Ventricular tachycardia and psychiatric depression in patients with coronary artery disease. Am J Med 1993;95: 23-8.

I I. Cavanaugh SVA. The prevalence of emotional and cognitive dysfunction in a general medical population: using the MMSE, GHQ, and BDI. Gen Hosp Psychiatry 1983; 5: 15-24.

12. Cavanaugh S, Clark D, Gibbons R. Diagnosing depression in the hospitalized medically ill. Psychosomatics 1983;24:809-15.

13. Cavanaugh S. Depression in the medically illclinical issues in diagnostic assesment. Psychosomatics 1995;36: 48-59.

14. Katon W \& Sullivan M. Depression and chronic medical illness. J Clin Psychiatry 1990; 5 I(6):3-II.

15. Verbosky L, Franco KN, Zrull JP. The relationship between depression and length of stay in the general hospital patient. J Clin Psychiatry 1993;54: 177-81.

16. Tufo H., Ostfeld A., Shekelle R. Central nervous system dysfunction following openheart surgery. JAMA 1 970;2 I 2: 1333-40.

17. Frasure-Smith N, Lesperance F, Talajic M. Depression following myocardial infarction: impact on 6-month survival. JAMA 1994;271:1080-82.

18. Silverstone PH. Depression and outcome in acute myocardial infarction. Br Med J I 987; 294: 219-20.

19. Carney RM, Rich MW, Freedland KE et al. Major depressive disorder predicts cardiac events in patients with coronary artery disease. Psychosom Med 1 988b; 50:627-33.

20. Carney RM, Rich MW, TeValde A et al. The relationship between, heart rate variability and depression in patients with coronay disease. J Psychosom Res 1988a; 32: 159-64.

21. Williams RB, ChesneyMA. Psychosocial Factors and Prognosis in Stablished Coronary Artery Disease. JAMA 1993;20(15):270.

22. Fielding R. Depression and acute myocardial infarction: a review and reinterpretation. Soc SciMed 1991; 32(9): 1017-28.

23. Hays RD, Wells KB, Sherbourne CD et al. Functioning and well-being outcomes of patients with depression compared with chronic general medical illness. Arch Gen Psychiatry 1995;52: I I-9.
24. Barefoot JC \& Scroll M. Symptoms of depression, acute myocardial infarction, and total mortality in a community sample. Circulation 1996; 93:1976-80.

25. Lesperance F, Frasure-Smith N, Talajic,M. Major Depression Before and After Myocardial Infarction: its nature and consequences. Psychosom Med I 996;58:99-I I 0.

26. Pratt LA, Ford D, Crum RM et al. Depression, psychotropic medication, and risk of miocardial infarction. Circulation 1996;94:3 I 23-29.

27. Appels A. Depression and coronary heart disease: observations and questions. J Psychossom Res 1997;43(5): 443-52.

28. Schwab JJ, Bialow M, Clemmons R, Martin $P$, Holzer CE. Diagnosing depression in medical inpatients. Acta Psychiatr Scand 1967; 43:255-66.

29. Moffic HS \& Paykel ES. Depression in medical inpatients. BrJ Psychiatry 1975; 126:346-53.

30. Plumb MM \& Holland J. Comparatice studies of psychologica function in patients with advanced cancer: I. Self-reported depressive symptoms. Psychosom Med 1977;39: 26476.

31. Clark DC, Cavanaugh SVA, Gibbons RD. The core symptoms of depression in medical and psychiatric patients. J Nerv Ment Dis 1983; I7I: 705-I3.

32. Willians JW Jr, Kerber CA, Mulrow CD, Medina A, Aguilar C. Depressive disorders in primary care: prevalence, functional disability, and identification. J Gen Intern Med 1995; 10(I):7-12.

33. Boland RJ, Diaz S, Lamdan RM, Ramchandani $D$, McCartneyJR. Overdiagnosis of depression in the general hospital. Gen Hosp Psychiatry 1996; I8(I):28-35.

34. Furlaneto LM. Diagnosticando depressão em pacientes internados em enfermarias de clínica médica. J Bras Psiq 1996; 45(6):363-70.

35. Wooley MA, Abins AL, Miranda K, Browner WS. Case-finding instruments for depression. Two questions are as good as many. J Gen Intern Med I 997; 12(7):439-45.

36. Gallo JJ, Rabins PV, Lyketsos CG, Tien AY, Anthony JC. Depression without sadness functional outcomes of nondysphoric depression in later life. J Am Geriatr Soc 1997; 45(5):570-8.

37.Schwab JJ, Bialow MR, Clemmons RS, Holzer CE. The affective symptomatology of depression in medical inpatients. Psychosomatics 1966; 7: 214-17.

Artigo recebido: 26/03/200 I Aceito para publicação: 03/07/200 I 